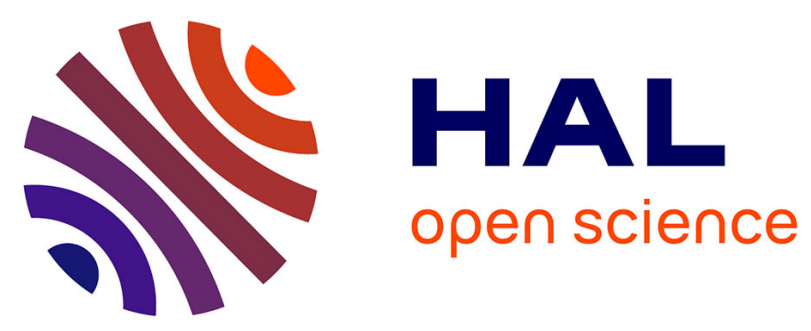

\title{
Ground state configurations of a simple cubic array of pseudo-spins $s=1 / 2$ with anisotropic exchange between nearest neighbours
}

\author{
E. Belorizky, R. Casalegno, P. Fries, J.J. Niez
}

\section{- To cite this version:}

E. Belorizky, R. Casalegno, P. Fries, J.J. Niez. Ground state configurations of a simple cubic array of pseudo-spins $\mathrm{s}=1 / 2$ with anisotropic exchange between nearest neighbours. Journal de Physique, 1978, 39 (7), pp.776-785. 10.1051/jphys:01978003907077600 . jpa-00208813

HAL Id: jpa-00208813

https://hal.science/jpa-00208813

Submitted on 1 Jan 1978

HAL is a multi-disciplinary open access archive for the deposit and dissemination of scientific research documents, whether they are published or not. The documents may come from teaching and research institutions in France or abroad, or from public or private research centers.
L'archive ouverte pluridisciplinaire HAL, est destinée au dépôt et à la diffusion de documents scientifiques de niveau recherche, publiés ou non, émanant des établissements d'enseignement et de recherche français ou étrangers, des laboratoires publics ou privés. 


\title{
GROUND STATE CONFIGURATIONS OF A SIMPLE CUBIC ARRAY OF PSEUDO-SPINS $S=\frac{1}{2}$ WITH ANISOTROPIC EXCHANGE BETWEEN NEAREST NEIGHBOURS
}

\author{
E. BELORIZKY, R. CASALEGNO \\ Laboratoire de Spectrométrie Physique $\left({ }^{*}\right)$ \\ Université Scientifique et Médicale de Grenoble, B.P. 53 X, 38041 Grenoble Cedex, France \\ P. FRIES and J. J. NIEZ (**) \\ Laboratoire de Chimie Physique Nucléaire, Département de Recherche Fondamentale, \\ Centre d'Etudes Nucléaires de Grenoble, B.P. 85 X, 38041 Grenoble Cedex, France
}

(Reçu le 20 janvier 1978, révisé le 28 février 1978, accepté le 13 mars 1978)

Résumé. - On cherche les configurations de plus basse énergie d'un système de pseudo-spins $S=1 / 2$ situés aux nœuds d'un réseau cubique simple, soumis à l'interaction la plus générale entre premiers voisins autorisée par la symétrie quaternaire de la liaison :

$$
\mathfrak{H}_{i j}=J_{\|} S_{i z} S_{j z}+J_{\perp}\left(S_{i x} S_{j x}+S_{i y} S_{j y}\right),
$$

pour une paire $(i, j)$ orientée selon $\mathrm{O} z$. En l'absence de champ extérieur, la configuration la plus stable est ferromagnétique lorsque $J_{\|}$et $J_{\perp}$ sont tous deux négatifs, et antiferromagnétique dans les autres cas ; dans cette dernière situation, on obtient différentes configurations antiferromagnétiques selon les signes de $J_{\|}$et $J_{\perp}$. A partir de tous ces configurations obtenues de manière classique, nous avons calculé le spectre d'ondes de spin afin de nous assurer de la-stabilité magnétique de ces systèmes et pour évaluer l'énergie quantique de l'état fondamental, ainsi que la déviation de spin moyenne dans cet état. Nous avons montré notamment que lorsque $J_{\|}=-J_{\perp}<0$, la configuration antiferromagnétique fondamentale est un état propre de l'Hamiltonien total. On établit ainsi un nouveau système magnétique à trois dimensions dont l'état fondamental est connu rigoureusement.

Abstract. - The configurations of lowest energy of a system of pseudo-spins $S=1 / 2$ forming a simple cubic lattice are analysed by considering the most general interaction, between nearest neighbours, allowed by a fourfold symmetry of the bond : $\mathbf{H}_{i j}=J_{\|} S_{i z} S_{j z}+J_{\perp}\left(S_{i x} S_{j x}+S_{i y} S_{j y}\right)$ for a pair $(i, j)$ oriented along the $z$ axis. In zero external field, the most stable configuration is either ferromagnetic when $J_{\|}$and $J_{\perp}$ are both negative, or antiferromagnetic in the other cases; in the latter situation various antiferromagnetic configurations are obtained depending on the signs of $J_{\|}$and $J_{\perp}$. For all these configurations, derived in a classical way, the spin wave spectrum is calculated in order to check the magnetic stability of the system as well as to evaluate the quantum ground state energy and the mean spin deviation in this ground state. It is also shown that when $J_{\|}=-J_{\perp}<0$, the antiferromagnetic ground configuration is an eigenstate of the total Hamiltonian. Thus a new three dimensional magnetic system, whose ground state is known exactly, is obtained.

1. Introduction. - In a previous paper [1] we studied the magnetic stability at $0 \mathrm{~K}$ of a simple cubic ferromagnetic array of pseudo-spins $S=1 / 2$ with anisotropic exchange between nearest neighbours.

(*) Laboratoire associé au C.N.R.S.

(**) Laboratoire de Spectrométrie Physique, B.P. 53 X, Grenoble également.
More precisely we considered a cubic crystal structure with magnetic ions having an odd number of electrons, forming a simple cubic array. This is the case of magnetic ions in the perovskite structure for example. If each magnetic ion is in a cubic crystal field, the single ion ground state is usually a $\Gamma_{6}$ or $\Gamma_{7}$ Kramers doublet which is assumed to be well separated from the other excited levels. This is a very common 
situation for rare-earth ions in a cubic field. We then introduce a pseudo-spin $S=1 / 2$, associated with each ground Kramers doublet, and the magnetic moment of the magnetic ion in its ground state is given by $\mathbf{M}=g \mu_{\mathbf{B}} \mathbf{S}$, where $g$ is isotropic [2]. It is very well known $[3,4]$ that the exchange interaction between these ions in their ground state will generally be anisotropic. Assuming this interaction to be small compared to the crystal field, and a $\mathrm{C}_{4 \mathrm{~h}}, \mathrm{C}_{4 \mathrm{v}}, \mathrm{D}_{4}$ or $\mathrm{D}_{4 \mathrm{~h}}$ symmetry of the bond joining two neighbouring ions, the most general form of the ion-ion exchange interaction allowed by this symmetry, expressed in terms of the pseudo-spin $S=1 / 2$, will be

$$
\mathcal{H}_{i j}=J_{\|} S_{i z} S_{j z}+J_{\perp}\left(S_{i x} S_{j x}+S_{i y} S_{j y}\right),
$$

for a pair $(i, j)$ of ions with their bond parallel to the $z$ axis. The corresponding form of $\mathcal{H}_{i j}$ for bonds parallel to the $x$ or $y$ axis are obtained from (1) by circular permutations of $x, y, z$.

In [1] we have shown that, in the limit of a zero external field, a ferromagnetic order is allowed at $0 \mathrm{~K}$ only for $J_{\|}$and $J_{\perp}$ both negative, with an easy direction of magnetization along a [100] axis. It is clear that, except for the special very simple case where

$$
J_{\|}=J_{\perp}<0
$$

(isotropic Heisenberg Hamiltonian), the classical ferromagnetic configuration with all the spins parallel to a [100] axis is not an eigenstate of the total Hamiltonian. However the diagonalization of the Hamiltonian of the problem, expressed in terms of quantum spin deviations from this configuration in the linearized Holstein-Primakoff formalism, leads to a new approximate quantum ground state whose energy $E_{0}^{\prime}$ is lower than the classical energy $E_{0}$, and whose excitations are the usual spin waves :

$$
\mathscr{H}=E_{0}^{\prime}+\sum_{\mathbf{k}} \lambda_{\mathbf{k}} \alpha_{\mathbf{k}}^{\dagger} \alpha_{\mathbf{k}} .
$$

The magnetic stability of the system is assured by the fact that, for all $\mathbf{k}, \lambda_{\mathbf{k}}$ is a real and positive quantity which simply means that the total energy of the system is not lowered by the excitation of spin waves.

A simple way for evaluating the difference between the new approximate ground state and the original classical state is given by the calculation of the mean spin deviation $\left\langle n_{i}\right\rangle$ from the classical state in the new state.

The aim of this article is the determination of the configurations of lowest energy for the same system of pseudo-spins $S=1 / 2$ forming a simple cubic lattice with the most general ion-ion interaction between nearest neighbours allowed by symmetry, for all possible values of $J_{\|}$and $J_{\perp}$.

If we rewrite $\mathscr{H}_{i, j}$ given by (1) as

$$
\mathscr{H}_{i j}=J_{\perp} \mathbf{S}_{i} \cdot \mathbf{S}_{j}+\left(J_{\|}-J_{\perp}\right) S_{i z} S_{j z},
$$

the total exchange Hamiltonian of our system of interacting spins is

$\mathscr{H}_{\mathrm{e}}=J_{\perp} \sum_{\substack{i<j \\ n . n .}} \mathbf{S}_{i} \cdot \mathbf{S}_{j}+\left(J_{\|}-J_{\perp}\right) \sum_{i} \sum_{r} S_{i}^{r} S_{i+\varepsilon_{r}}^{r}$

where the summation $i$ is taken over the $N$ equivalent ions of the sample, and $r$ stands for $x, y, z$; for example $i+\varepsilon_{z}$ is the neighbour of the spin $i$ located in the positive $z$ direction at a distance $(0,0, a), a$ being the cubic lattice parameter.

There exists no rigorous theory for the prediction of the ordered structures corresponding to a given Hamiltonian, and approximations must be used. In section 2 we shall follow the classical method of Yoshimori [5]. Then for each configuration of lowest energy associated with each possible sign and magnitude of $J_{\|}$and $J_{\perp}$, we shall calculate in section 3 the spin wave spectra in order to check the magnetic stability of the system. From the dispersion relations we shall be able to evaluate, in section 4 , the quantum ground state energies and the mean spin deviations in these states. The new phase diagram will be extensively discussed in section 5 .

2. Classical approach. - For a while we shall treat the spins as classical vectors and look for the minimum of $\mathcal{H}$ given by equation (4). For this purpose we perform the Fourier transformations on the components $S_{i}^{r}$ of $\mathbf{S}_{i}$ which are real vectors :

$S_{i}^{r}=\sum_{\mathbf{k}^{r}} S^{r}\left(\mathbf{k}^{r}\right) \mathrm{e}^{i \mathbf{k}^{r} \cdot \mathbf{R}_{i}} \quad$ with $\quad S^{r}\left(\mathbf{k}^{r}\right)=S^{r^{*}}\left(-\mathbf{k}^{r}\right)$,

where the three vectors $\mathbf{k}^{r}$ run over $N$ wave vectors in the Brillouin zone corresponding to the cubic unit cell. Then we have

$$
\mathscr{H}=N \sum_{r} \sum_{\mathbf{k}^{r}} S^{r}\left(\mathbf{k}^{r}\right) S^{r}\left(-\mathbf{k}^{r}\right) W_{\mathbf{k}^{r}}^{r},
$$

where

$$
\begin{aligned}
& W_{\mathbf{k}^{x}}^{x}=J_{\|} \cos k_{x}^{x} a+J_{\perp}\left(\cos k_{y}^{x} a+\cos k_{z}^{x} a\right), \\
& W_{\mathbf{k}^{y}}^{y}=J_{\|} \cos k_{y}^{y} a+J_{\perp}\left(\cos k_{z}^{y} a+\cos k_{x}^{y} a\right), \\
& W_{\mathbf{k}^{z}}^{z}=J_{\|} \cos k_{z}^{z} a+J_{\perp}\left(\cos k_{x}^{z} a+\cos k_{y}^{z} a\right) .
\end{aligned}
$$

In these notations $k_{\alpha}^{r}$ is the $\alpha$ component of the $\mathbf{k}^{r}$ vector relative to the $r$ component of the spins.

Since we must have

we get

$$
\mathbf{S}_{i}^{2}=S^{2},
$$

$$
\sum_{r} \sum_{\mathbf{k}^{r}, \mathbf{k}^{\prime r}} S^{r}\left(\mathbf{k}^{r}\right) S^{r}\left(\mathbf{k}^{\prime r}\right) \mathrm{e}^{i\left(\mathbf{k}^{r}+\mathbf{k}^{\prime r}\right) \cdot \mathbf{R}_{i}}=S^{2} .
$$

The stable structure is that which minimizes the energy (6), while satisfying the $N$ strong constraints (8) or (9). This problem may be formally solved by using a Lagrange-multiplier for each spin but this is an impossible task. Instead of that, following Yoshimori [5] or 
Luttinger [6], we minimize the energy under the much less stringent condition deduced from (8) :

$$
\sum_{i} \mathbf{S}_{i}^{2}=N S^{2},
$$

which may be rewritten

$$
\sum_{r} \sum_{\mathbf{k}^{r}} S^{r}\left(\mathbf{k}^{r}\right) S^{r}\left(-\mathbf{k}^{r}\right)=S^{2} .
$$

If the solution which is obtained in this way happens to satisfy also the strong constraints (8), we shall have our stable magnetic configuration.

The minimization of the total energy (6) under the weak constraint (10) reads

$$
\mathrm{d} \mathscr{C}=N \lambda \mathrm{d} S^{2},
$$

where $\lambda$ is the unique Lagrange multiplier of the problem. The $3 N S^{r}\left(k^{r}\right)$ being considered in (12) as independent variables, we get immediately from (6) and (11)

$$
\lambda=W_{\mathbf{k}^{r}}^{r},
$$

where the $W_{\mathbf{k}^{x}}^{x}, W_{\mathbf{k}^{y}}^{y}, W_{\mathbf{k}^{z}}^{z}$ are defined by (7). Now if the solution (13) is put into (6), we get from (11) :

$$
\mathscr{H}=N \lambda S^{2},
$$

and it is seen that $\mathcal{H}$ is minimum when $\lambda$ is minimum. It is now possible to investigate the ground magnetic configurations for all possible values of $J_{\|}$and $J_{\perp}$ by looking at equation (7) :

$2.1 J_{\|}<0, J_{\perp}<0$. . The minimum value of $\lambda$ is given by $\left(J_{\|}+2 J_{\perp}\right)$ for $\mathbf{k}^{x}=\mathbf{k}^{y}=\mathbf{k}^{z}=0$. The spin configuration is given by (5) :

$$
\mathbf{S}_{i}=\mathbf{S}(0) \text {. }
$$

Obviously, for this solution, the weak condition (10) which is expressed by $\mathbf{S}(0)^{2}=S^{2}$ is identical to the strong condition (8). This is the ferromagnetic configuration studied in the previous paper [1] and we get the same condition for it : $J_{\|}$and $J_{\perp}$ must be both negative. All the spins are parallel and may point in an arbitrary direction. For the sake of simplicity we shall assume that we have a vanishingly small anisotropy field in the [001] direction taken as $\mathrm{z}$ axis. We thus get the configuration called $Z_{1}$ represented in figure 1 . Otherwise the classical ferromagnetic configuration is a linear combination of $X_{1}, Y_{1}, Z_{1}$, where $X_{1}$ and $Y_{1}$ are obtained from $Z_{1}$ through cubic rotations. It must be recalled at this point that the quantum approach of

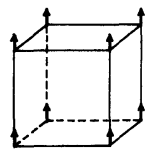

(a)

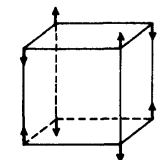

(b)

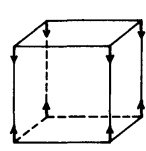

(c)

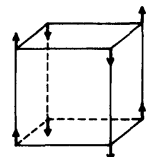

(d) $Z_{5}: J_{/ 1}<0, J_{1}>0$

the ferromagnetic configuration leads to an anisotropic ground state energy, but still the easy magnetization axes are the fourfold axes of the cube [1].

$2.2 J_{\|}>0, J_{\perp}>0$. - The minimum value of $\lambda$ is $-\left(J_{\|}+2 J_{\perp}\right)$ for

$$
\mathbf{k}^{x}=\mathbf{k}^{y}=\mathbf{k}^{z}=\mathbf{q},
$$

the components of the wave vector $\mathbf{q}$ being $(\pi / a$, $\pi / a, \pi / a)$.

From (5) the spin configuration is given by

$$
\mathbf{S}_{i}=(-1)^{l_{l}+m_{i}+n_{i}} \mathbf{S}(\mathbf{q}),
$$

where $\mathbf{q}$ is defined by (16) and $l_{i} a, m_{i} a, n_{i} a$ are the components of $\mathbf{R}_{i}$ in a system of fourfold axes with one spin site taken as origin.

Here again it is easy to check that the solution (17) which satisfies the weak constraint (11) rewritten now as

$$
\mathbf{S}^{2}(\mathbf{q})=S^{2}
$$

(for $\mathbf{q}$ given by (16), $\mathbf{S}(-\mathbf{q})=\mathbf{S}(\mathbf{q})$ ), also satisfies the strong constraint (9) which is identical to (18).

In this case the classical ground configuration is such that each spin is surrounded by six nearest neighbours with opposite direction. Still assuming that we have a vanishingly small anisotropy field in the $z$ direction, we get the configuration called $Z_{8}$ represented in figure 1. Otherwise the classical antiferromagnetic ground configuration is a linear combination of $\mathrm{X}_{8}, \mathrm{Y}_{8}, \mathrm{Z}_{8}$.

$2.3 J_{\|}>0, J_{\perp}<0 .-$ The minimum value of $\lambda$ is $-J_{\|}+2 J_{\perp}$ and is obtained when the $\mathbf{k}^{r}$ have the following components :

$$
\begin{aligned}
\mathbf{k}^{x} & \equiv(\pi / a, 0,0), \\
\mathbf{k}^{y} & \equiv(0, \pi / a, 0), \\
\mathbf{k}^{z} & =(0,0, \pi / a) .
\end{aligned}
$$

From (5) the spin configuration is given by

$$
\begin{aligned}
& S_{i}^{x}=(-1)^{l_{i}} S^{x}\left(\mathbf{k}^{x}\right), \\
& S_{i}^{y}=(-1)^{m_{i}} S^{y}\left(\mathbf{k}^{y}\right), \\
& S_{i}^{z}=(-1)^{n_{i}} S^{z}\left(\mathbf{k}^{z}\right) .
\end{aligned}
$$

The weak and strong constraints (10) and (8) are still identical and are expressed by

$$
\left[S^{x}\left(\mathbf{k}^{x}\right)\right]^{2}+\left[S^{y}\left(\mathbf{k}^{y}\right)\right]^{2}+\left[S^{z}\left(\mathbf{k}^{z}\right)\right]^{2}=S^{2},
$$

where the $\mathbf{k}^{r}$ are defined by (19).

We get a rather complicated structure which, however, may be easily visualized by considering that it is a linear combination of the three configurations $\mathrm{X}_{2}, \mathrm{Y}_{2}, \mathrm{Z}_{2}$, where $\mathrm{Z}_{2}$, for instance, corresponds to $S_{i}^{x}=S_{i}^{y}=0$ and $S_{i}^{z}=(-1)^{n_{i}} S^{z}\left(\mathbf{k}^{z}\right)=(-1)^{n_{i}} S$. This configuration shows alternate $(001)$ planes with

FIG. 1. - The four ground configurations corresponding to the Hamiltonian (6). 
spins up and down successively. This is the configuration obtained with a vanishingly small anisotropy field in the $z$ direction and it is represented in figure 1 .

$2.4 J_{\|}<0, J_{\perp}>0$. - This situation is very similar to the previous one; the minimum value of $\lambda$ is $J_{\|}-2 J_{\perp}$, and this is realized when the $\mathbf{k}^{r}$ have the following components :

$$
\begin{aligned}
& \mathbf{k}^{x} \equiv(0, \pi / a, \pi / a), \\
& \mathbf{k}^{y} \equiv(\pi / a, 0, \pi / a), \\
& \mathbf{k}^{z} \equiv(\pi / a, \pi / a, 0) .
\end{aligned}
$$

The spin configuration is given by

$$
\begin{aligned}
& S_{i}^{x}=(-1)^{m_{i}+n_{i}} S^{x}\left(\mathbf{k}^{x}\right), \\
& S_{i}^{y}=(-1)^{n_{i}+l_{i}} S^{y}\left(\mathbf{k}^{y}\right), \\
& S_{i}^{z}=(-1)^{l_{i}+m_{i}} S^{z}\left(\mathbf{k}^{z}\right) .
\end{aligned}
$$

The weak and strong constraints are identical and are still expressed by (21) with the $\mathbf{k}^{r}$ given by (22).

The structure is a linear combination of the three configurations $\mathrm{X}_{5}, \mathrm{Y}_{5}, \mathrm{Z}_{5}$ where $\mathrm{Z}_{5}$, for instance, corresponds to $S_{i}^{x}=S_{i}^{y}=0$ and

$$
S_{i}^{z}=(-1)^{l_{i}+m_{i}} S^{z}\left(\mathbf{k}^{z}\right)=(-1)^{l_{i}+m_{i}} S .
$$

This configuration is such that we have a vertical line with parallel spins up and parallel vertical lines at distance $a$ with spins down. This structure is represented in figure 1 .

These notations $Z_{1}, Z_{2}, Z_{5}, Z_{8}$ are those introduced by Luttinger and Tisza [7] for dipolar interactions. Indeed, these configurations could have been obtained very simply by considering only spins pointing along the $z$ direction and by limiting ourselves to arrays such that two spins separated by a distance $2 a$ along the $x$, or $y$, or $z$ directions are parallel ( $\Gamma^{2}$ class array). In these conditions, starting from the spin $\mathbf{S}_{0}$ at the origin, the whole simple cubic crystal structure may be generated by the three primitive translations $\mathbf{i} a, \mathbf{j} a, \mathbf{k} a$ where $\mathbf{i}, \mathbf{j}, \mathbf{k}$ are unit vectors in the $x, y, z$ directions. Then, if $\mathbf{S}_{0}$ is a spin up, there are eight different configurations corresponding to the orientation up or down of the three nearest neighbours $\mathbf{S}_{1}, \mathbf{S}_{2}, \mathbf{S}_{3}$ located at $(a, 0,0)$, $(0, a, 0),(0,0, a)$. The four configurations $\mathrm{Z}_{1}, \mathrm{Z}_{8}, \mathrm{Z}_{5}, \mathrm{Z}_{2}$

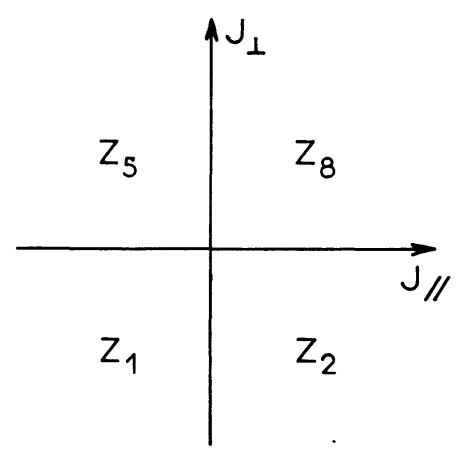

Fig. 2. - Classifical ground configurations for all possible values of $J_{\|}$and $J_{\perp}$. are four of these eight possibilities which are shown to minimize the energy in each particular case. The advantage of the method of Yoshimori is that it does not exclude $a$ priori configurations different from the class $\Gamma^{2}$ and that all possible configurations are investigated including for example helimagnetic solutions.

The various results obtained in this section are summarized in figure 2 which represents the classical phase diagram. In all the cases the classical ground state energy $E_{0}$ expressed in terms of the parameter $t=J_{\|} / J_{\perp}$ is given by

$$
\begin{aligned}
E_{0}=-N S^{2}\left(\left|J_{\|}\right|\right. & \left.+2\left|J_{\perp}\right|\right)= \\
& =-N S^{2}\left|J_{\perp}\right|(|t|+2) .
\end{aligned}
$$

For what follows, an important remark must be made. The configurations $Z_{5}$ and $Z_{2}$ may be deduced from the configurations $Z_{1}$ and $Z_{8}$ in a very simple way. Let $x, y, z$ be the fourfold axes associated with the spin which is at the origin, we associate to a spin located in $\mathbf{R}_{i}$ a local system of axes $x_{i}, y_{i}, z_{i}$ such that

$$
\begin{aligned}
& x_{i}=(-1)^{m_{i}+n_{i}} x, \\
& y_{i}=(-1)^{n_{i}+l_{i}} y, \\
& z_{i}=(-1)^{l_{i}+m_{i}} z .
\end{aligned}
$$

We get in this way four kinds of orthogonal systems of axes which are always direct.

Now if we consider two neighbouring interacting ions, the two local systems of axes defined by (25) and associated with each of the spins will have parallel axes in the direction of the bond and opposite axes in the two other directions perpendicular to the bond. This procedure is equivalent to keeping $J_{\|}$and changing the sign of $J_{\perp}$ in the exchange Hamiltonian.

Now for $J_{\|}<0$, if we start from the $\mathrm{Z}_{1}$ configuration and use this procedure, i.e. of changing $J_{\perp}$ into $-J_{\perp}$, we get the $Z_{5}$ configuration as can be easily seen from figure 1 because the spins along a vertical line remain parallel, and neighbouring spins in a horizontal plane which are parallel in $Z_{1}$ become antiparallel in $Z_{5}$. So $Z_{5}$ is simply the ferromagnetic configuration $Z_{1}$ in which we associate the local axes (25) with each lattice site.

Similarly for $J_{\|}>0$, it is easy to check that $Z_{2}$ is simply deduced from $Z_{8}$ by changing $J_{\perp}$ into $-J_{\perp}$, which means that the antiferromagnetic configuration $Z_{2}$ is simply $Z_{8}$ in which we take the local axes (25).

We could also deduce $Z_{2}$ from $Z_{1}$ by changing $J_{\|}$ into $-J_{\|}$and keeping $J_{\perp}$, a procedure which is equivalent to introducing a local system of axes defined by

$$
\begin{aligned}
x_{i} & =(-1)^{l_{i}} x, \\
y_{i} & =(-1)^{m_{i}} y, \\
z_{i} & =(-1)^{n_{i}} z .
\end{aligned}
$$


However this transformation makes use of inverse local frames and will not be as helpful for the quantum approach to the problem.

Obviously the transformations (25) and (26) are simply correlated with (23) and (20).

It may be of interest to give the magnetic point group associated to each of the above magnetic structure. The magnetic point group of $Z_{1}$ is $D_{4 h}\left(C_{4 h}\right)$ where $C_{4 h}$ is the invariant unitary subgroup [8], and the magnetic point group of $Z_{2}, Z_{5}$ and $Z_{8}$ is in each case $D_{4 h} \times T$, where $T$ is the time reversal operator.

It may be noted that the four structures $Z_{3}, Z_{4}, Z_{6}$ and $Z_{7}$ described by Luttinger and Tisza [7] have only a magnetic point group $\mathrm{D}_{2 \mathrm{~h}} \times T$. Thus the classical ground configuration is always associated in our problem with a higher symmetry of the magnetic point group.

2.5 SPeCIAL CASES : $J_{\|}$OR $J_{\perp}=0 .-2.5 .1 J_{\|}=0$. - We first restrict ourselves to configurations where the spins are along the $z$ axis. When $J_{\perp}<0$, the ground configurations $Z_{1}$ and $Z_{2}$ have the same.energy, and similarly when $J_{\perp}>0$, the ground configurations $Z_{5}$ and $Z_{8}$ have the same energy. More generally, in this case, there is no correlation between the orientation of the spins of two adjacent planes perpendicular to the $z$ axis. If $J_{\perp}<0$ each of these planes is a ferromagnetic layer; $Z_{1}$ and $Z_{2}$ are simply two particular cases where two adjacent planes have their spins parallel or antiparallel respectively. Similarly, if $J_{\perp}>0$, each plane perpendicular to the $z$ axis is an antiferromagnetic layer; $Z_{5}$ and $Z_{8}$ are still two particular configurations where two neighbouring ions on the $z$ axis have their spins parallel or antiparallel.

The most general configuration is obtained by combining linearly the above configurations where the spins are along the $z$ axis, with the equivalent configurations where they point along the $x$ and $y$ axes.

2.5.2 $J_{\perp}=0$. - As before, if we restrict ourselves, as a first step, to configurations where the spins are along the $z$ axis, it can easily be seen that we get configurations with vertical lines, such that there is no correlation between the orientation of the spins of two adjacent vertical lines. If $J_{\|}<0$, these lines are ferromagnetic, and if $J_{\|}>0$, they are antiferromagnetic. $\mathrm{Z}_{1}, \mathrm{Z}_{5}$ or $\mathrm{Z}_{3}$ and $\mathrm{Z}_{4}$ (the two latter configurations are not represented in figure 1 , but are given in ref. [7]) are particular configurations corresponding to ferromagnetic vertical lines and have all the same energy $N S^{2} J_{\|}$. Similarly $\mathrm{Z}_{2}, \mathrm{Z}_{8}, \mathrm{Z}_{6}$ and $\mathrm{Z}_{7}$ (see ref. [7]) are particular configurations corresponding to antiferromagnetic vertical lines and have the same energy $-N S^{2} J_{\|}$.

The most general configuration is rather complicated in this case and is obtained by linear combination of these configurations with the equivalent ones whose spins point along the $x$ or $y$ axes. We thus get a very high degeneracy, and the classical structure may be very complicated. We would like to show that, for example, we can have helicoidal solutions.

When $J_{\|}<0, \lambda$ is minimum and equal to $J_{\|}$for the following components of $\mathbf{k}^{x}, \mathbf{k}^{y}, \mathbf{k}^{z}$, as can be checked from (7) :

$$
\begin{aligned}
& \mathbf{k}^{x}=\left(0, k_{y}^{x}, k_{z}^{x}\right), \\
& \mathbf{k}^{y}=\left(k_{x}^{y}, 0, k_{z}^{y}\right), \\
& \mathbf{k}^{z}=\left(k_{x}^{z}, k_{y}^{z}, 0\right) .
\end{aligned}
$$

The non-zero components of (27) are a priori arbitrary. Let us choose the particular solution given by :

$$
k_{y}^{x}=k_{x}^{y}=k_{x}^{z}=k_{y}^{z}=0 ; \quad k_{z}^{x}=k_{z}^{y}=q \neq 0 .
$$

The weak condition (11) may be written as :

$2 S_{x}(q) S_{x}(-q)+2 S_{y}(q) S_{y}(-q)+S_{z}^{2}(0)=S^{2}$,

and the strong condition (9) becomes

$$
\begin{aligned}
{\left[S^{x}(q)\right]^{2} } & \mathrm{e}^{2 i q R_{i z}}+\left[S^{x}(-q)\right]^{2} \mathrm{e}^{-2 i q R_{i z}}+ \\
& +2 S^{x}(q) S^{x}(-q)+\left[S^{y}(q)\right]^{2} \mathrm{e}^{2 i q R_{i z}} \\
& +\left[S^{y}(-q)\right]^{2} \mathrm{e}^{-2 i q R_{i z}} \\
& +2 S^{y}(q) S^{y}(-q)+\left[S^{z}(0)\right]^{2}=S^{2} .
\end{aligned}
$$

The strong condition will satisfy the weak condition if

$$
\left[S^{x}(q)\right]^{2}+\left[S^{y}(q)\right]^{2}=0 .
$$

From (28) and (30) we get

$$
\mathbf{S}(q)=\left(\mathbf{u}_{1}+i \mathbf{u}_{2}\right) A S,
$$

where $\mathbf{u}_{1}$ and $\mathbf{u}_{2}$ are the unit vectors along the $x$ and $y$ directions, and $A$ is a normalization constant given by

$$
A=\frac{1}{2} \sqrt{1-\left[\frac{S_{z}(0)}{S}\right]^{2}} .
$$

From (5) we get the helimagnetic structure

$\mathbf{S}_{i}=2 A S\left[\cos \left(q R_{i z}\right) \mathbf{u}_{1}-\sin \left(q R_{i z}\right) \mathbf{u}_{2}\right]+S_{z}(0) \mathbf{u}_{3}$.

3. Quantum approach. Spin wave spectrum. Starting from each of the configurations described in figure 1 , we shall calculate the spin wave spectrum corresponding to a Hamiltonian including the exchange interaction and a vanishingly small anisotropy field $H_{a}$ along the $z$ direction in order to privilege this direction. This calculation will be performed in the framework of the linearized Holstein-Primakoff formalism.

For $Z_{1}$, this calculation was made in our previous 
paper [1] and the total Hamiltonian could be written in the form (2) where $\lambda_{\mathbf{k}}$ and $E_{0}^{\prime}$ are given by

$$
\begin{aligned}
& \lambda_{\mathbf{k}}=4 S\left|J_{\perp}\right|\left[\left(t \sin ^{2} \alpha+\sin ^{2} \beta+\sin ^{2} \gamma-\frac{H_{a}}{4 S J_{\perp}}\right) \times\right. \\
& \left.\times\left(\sin ^{2} \alpha+t \sin ^{2} \beta+\sin ^{2} \gamma-\frac{H_{a}}{4 S J_{\perp}}\right)\right]^{1 / 2} \\
& E_{0}^{\prime}=-N S(S+1)\left|J_{\perp}\right|(2+t)+\frac{1}{2} \sum_{\mathbf{k}} \lambda_{\mathbf{k}}
\end{aligned}
$$

where

$$
t=\frac{J_{\|}}{J_{\perp}} ; \quad \alpha=\frac{k_{x} a}{2}, \quad \beta=\frac{k_{y} a}{2}, \quad \gamma=\frac{k_{z} a}{2} .
$$

Here $J_{\|}$and $J_{\perp}$ are both negative, thus $t>0$. The expression (33) is easily deduced from equation (21) of reference [1] by taking $l=m=0, n=1$ and $H_{a}=g \mu_{\mathrm{B}} H$ (the anisotropy field or external magnetic field along the $z$ direction). The summation in (34) is taken over the Brillouin zone, i.e. $-\frac{\pi}{2}<\alpha, \beta, \gamma \leqslant \frac{\pi}{2}$. Typical dispersion curves corresponding to relation (33) in the limit where $H_{a}=0$ are represented in figure $4 a$. For $\mathbf{k} / /[001], \lambda_{\mathbf{k}} /\left(4 S\left|J_{\perp}\right|\right)$ is independent of $t$, but for any other direction of $\mathbf{k}$ we get diagrams very similar to the one given here for $\mathbf{k} / /[100]$. It should be noted that as for a Heisenberg ferromagnet $(t=1), \lambda_{\mathbf{k}} \rightarrow 0$ when $\mathbf{k} \rightarrow 0$ when $t \neq 1$. As will be discussed later this result is true only in the framework of the linearized Holstein-Primakoff formalism.

For the three antiferromagnetic configurations $Z_{8}$, $\mathrm{Z}_{2}$ and $\mathrm{Z}_{5}$ we start from the total Hamiltonian

$$
\begin{aligned}
\mathcal{H}= & J_{\perp}\left(\sum_{\substack{i<i^{\prime} \\
n . n .}} \mathbf{S}_{i} \cdot \mathbf{S}_{i^{\prime}}+\sum_{\substack{j<j^{\prime} \\
n . n .}} \mathbf{S}_{j} \cdot \mathbf{S}_{j^{\prime}}+\sum_{\substack{i, j \\
n, n .}} \mathbf{S}_{i} \cdot \mathbf{S}_{j}\right)+ \\
& +\left(J_{\|}-J_{\perp}\right)\left(\sum_{i, r} S_{i}^{r} S_{i+\varepsilon_{r}}^{r}+\sum_{j, r} S_{j}^{r} S_{j+\varepsilon_{r}}^{r}+\right. \\
& \left.+\sum_{r} S_{i}^{r} S_{j_{r}}^{r}+\sum_{r} S_{j}^{r} S_{i_{r}}^{r}\right)-H_{a} \sum_{i} S_{i}^{z}+H_{a} \sum_{j} S_{j}^{z},
\end{aligned}
$$

where the indices $i$ and $j$ refer to the different sublattices with spins up and down respectively. All the exchange interactions are taken between nearest neighbours exclusively; $i+\varepsilon_{r}$ is the neighbour of the spin $i$ belonging to the same sublattice in the positive $r$ direction and $j_{r}$ is the neighbour of $i$ belonging to the opposite sublattice in the positive $r$ direction. $H_{a}$ is a positive anisotropy field. Introducing the magnon creation and annihilation operators

$a_{\mathbf{k}}=\sqrt{\frac{2}{N}} \sum_{i} \mathrm{e}^{i \mathbf{k} \cdot \mathbf{R}_{i}} a_{i}, \quad b_{\mathbf{k}}=\sqrt{\frac{2}{N}} \sum_{j} \mathrm{e}^{-i \mathbf{k} \cdot \mathbf{R}_{j}} b_{j}$,

we get a Hamiltonian which is quadratic with respect to the Bose operators and which has the following general form :

$$
\begin{aligned}
& \mathcal{H}=E_{0}+\sum_{\mathbf{k}} \omega_{\mathbf{k}}\left(a_{\mathbf{k}}^{\dagger} a_{\mathbf{k}}+b_{\mathbf{k}}^{\dagger} b_{\mathbf{k}}\right)+ \\
& +\sum_{\mathbf{k}} \gamma_{\mathbf{k}}\left(a_{\mathbf{k}} a_{-\mathbf{k}}+a_{\mathbf{k}}^{\dagger} a_{-\mathbf{k}}^{\dagger}+b_{\mathbf{k}} b_{-\mathbf{k}}+b_{\mathbf{k}}^{\dagger} b_{-\mathbf{k}}^{\dagger}\right) \\
& +\sum_{\mathbf{k}} \delta_{\mathbf{k}}\left(a_{\mathbf{k}} b_{\mathbf{k}}+a_{\mathbf{k}}^{\dagger} b_{\mathbf{k}}^{\dagger}\right)+\sum_{\mathbf{k}} \varepsilon_{\mathbf{k}}\left(a_{\mathbf{k}} b_{-\mathbf{k}}^{\dagger}+a_{\mathbf{k}}^{\dagger} b_{-\mathbf{k}}\right),
\end{aligned}
$$

where $E_{0}$ is the classical ground state energy given by (24) and $\omega_{\mathbf{k}}, \gamma_{\mathbf{k}}, \delta_{\mathbf{k}}$ and $\varepsilon_{\mathbf{k}}$ are real even functions of $\mathbf{k}$ which will be given later for each specific case. The summation over $\mathbf{k}$ is taken over the first Brillouin zone of the magnetic sublattice.

The Hamiltonian (38) may be diagonalized with the help of the following linear transformation :

$$
\begin{aligned}
& \alpha_{\mathbf{k}}=t_{\mathbf{k}} a_{\mathbf{k}}+u_{\mathbf{k}} a_{-\mathbf{k}}^{\dagger}+v_{\mathbf{k}} b_{-\mathbf{k}}+w_{\mathbf{k}} b_{\mathbf{k}}^{\dagger}, \\
& \beta_{\mathbf{k}}=t_{\mathbf{k}}^{\prime} a_{\mathbf{k}}+u_{\mathbf{k}}^{\prime} a_{-\mathbf{k}}^{\dagger}+v_{\mathbf{k}}^{\prime} b_{-\mathbf{k}}+w_{\mathbf{k}}^{\prime} b_{+\mathbf{k}}^{\dagger},
\end{aligned}
$$

where $t_{\mathbf{k}}, u_{\mathbf{k}}, v_{\mathbf{k}}, w_{\mathbf{k}}, t_{\mathbf{k}}^{\prime}, u_{\mathbf{k}}^{\prime}, v_{\mathbf{k}}^{\prime}, w_{\mathbf{k}}^{\prime}$ are real even functions of $\mathbf{k}$ related by

$$
\begin{aligned}
t_{\mathbf{k}}^{2}-u_{\mathbf{k}}^{2}+v_{\mathbf{k}}^{2}-w_{\mathbf{k}}^{2} & =1, \\
t_{\mathbf{k}}^{\prime 2}-u_{\mathbf{k}}^{\prime 2}+v_{\mathbf{k}}^{\prime 2}-w_{\mathbf{k}}^{\prime 2} & =1,
\end{aligned}
$$

because the new creation and annihilation operators $\alpha_{\mathbf{k}}, \alpha_{\mathbf{k}}^{\dagger}, \beta_{\mathbf{k}}, \beta_{\mathbf{k}}^{\dagger}$ must satisfy the usual commutation rules of Bose operators :

$$
\left[\alpha_{\mathbf{k}}, \alpha_{\mathbf{k}}^{\dagger}\right]=\left[\beta_{\mathbf{k}}, \beta_{\mathbf{k}}^{\dagger}\right]=1 .
$$

Using expressions (39) and (40) we obtain

$$
\mathscr{H}=E_{0}^{\prime}+\sum_{\mathbf{k}} \lambda_{\mathbf{k}} \alpha_{\mathbf{k}}^{\dagger} \alpha_{\mathbf{k}}+\sum_{\mathbf{k}} \mu_{\mathbf{k}} \beta_{\mathbf{k}}^{\dagger} \beta_{\mathbf{k}},
$$

where

$$
\begin{aligned}
& \lambda_{\mathbf{k}}=\left[\left(\omega_{\mathbf{k}}+\varepsilon_{\mathbf{k}}\right)^{2}-\left(\delta_{\mathbf{k}}+2 \gamma_{\mathbf{k}}\right)^{2}\right]^{1 / 2} \\
& \mu_{\mathbf{k}}=\left[\left(\omega_{\mathbf{k}}-\varepsilon_{\mathbf{k}}\right)^{2}-\left(\delta_{\mathbf{k}}-2 \gamma_{\mathbf{k}}\right)^{2}\right]^{1 / 2},
\end{aligned}
$$

and

$$
E_{0}^{\prime}=E_{0}+\frac{1}{2} \sum_{\mathbf{k}}\left(\lambda_{\mathbf{k}}+\mu_{\mathbf{k}}-2 \omega_{\mathbf{k}}\right)
$$

The expression of the coefficients introduced in (39) which realize this diagonalization are

$$
\begin{gathered}
t_{\mathbf{k}}=v_{\mathbf{k}}=\frac{2 \gamma_{\mathbf{k}}+\delta_{\mathbf{k}}}{\left[4 \lambda_{\mathbf{k}}\left(\omega_{\mathbf{k}}+\varepsilon_{\mathbf{k}}-\lambda_{\mathbf{k}}\right)\right]^{1 / 2}} \\
u_{\mathbf{k}}=w_{\mathbf{k}}=\left[\frac{\omega_{\mathbf{k}}+\varepsilon_{\mathbf{k}}-\lambda_{\mathbf{k}}}{4 \lambda_{\mathbf{k}}}\right]^{1 / 2} \\
t_{\mathbf{k}}^{\prime}=-v_{\mathbf{k}}^{\prime}=\frac{-2 \gamma_{\mathbf{k}}+\delta_{\mathbf{k}}}{\left[4 \mu_{\mathbf{k}}\left(\omega_{\mathbf{k}}-\varepsilon_{\mathbf{k}}-\mu_{\mathbf{k}}\right)\right]^{1 / 2}} \\
u_{\mathbf{k}}^{\prime}=-w_{\mathbf{k}}^{\prime}=\left[\frac{\omega_{\mathbf{k}}-\varepsilon_{\mathbf{k}}-\mu_{\mathbf{k}}}{4 \mu_{\mathbf{k}}}\right]^{1 / 2} .
\end{gathered}
$$

The spin wave spectra of the three antiferromagnetic configurations studied in the: previous section are now examined as particular cases of the above general equations. 
3.1 Configuration $Z_{8}$. - Each magnetic sublattice is a face centred cubic lattice (each cube being of side $2 a$ ) and the corresponding first Brillouin zone in the reciprocal space which is the Wigner-Seitz unit cell of a cubic centred lattice [9] is represented in figure $3 a$. In this case the spin wave Hamiltonian (38) is such that $\omega_{\mathbf{k}}=2 S\left(2 J_{\perp}+J_{\|}\right)+H_{a}$,

$$
\begin{aligned}
& \gamma_{\mathbf{k}}=0, \\
& \delta_{\mathbf{k}}=S\left(J_{\perp}+J_{\|}\right)\left(\cos k_{x} a+\cos k_{y} a\right)+2 S J_{\perp} \cos k_{z} a, \\
& \varepsilon_{\mathbf{k}}=S\left(J_{\|}-J_{\perp}\right)\left(\cos k_{x} a+\cos k_{y} a\right) .
\end{aligned}
$$

From (43) and (46) we may deduce the dispersion relations $\lambda_{\mathbf{k}}$ and $\mu_{\mathbf{k}}$ which may be rewritten, by using the notations introduced in (35), as

$$
\begin{aligned}
& \lambda_{\mathbf{k}}=4 S\left|J_{\perp}\right|\left[\left(t \cos ^{2} \alpha+\cos ^{2} \beta+\cos ^{2} \gamma+\frac{H_{a}}{4 S J_{\perp}}\right)\left(\sin ^{2} \alpha+t \sin ^{2} \beta+\sin ^{2} \gamma+\frac{H_{a}}{4 S J_{\perp}}\right)\right]^{1 / 2}, \\
& \mu_{\mathbf{k}}=4 S\left|J_{\perp}\right|\left[\left(t \sin ^{2} \alpha+\sin ^{2} \beta+\sin ^{2} \gamma+\frac{H_{a}}{4 S J_{\perp}}\right)\left(\cos ^{2} \alpha+t \cos ^{2} \beta+\cos ^{2} \gamma+\frac{H_{a}}{4 S J_{\perp}}\right)\right]^{1 / 2},
\end{aligned}
$$

with

$$
-\frac{\pi}{2}<\alpha, \beta, \gamma \leqslant \frac{\pi}{2} \quad \text { and } \quad|\alpha|+|\beta|+|\gamma| \leqslant \frac{3 \pi}{4} .
$$
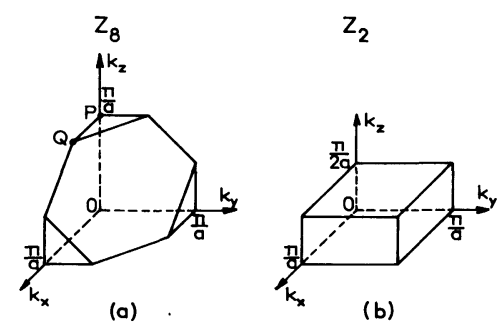

(b)

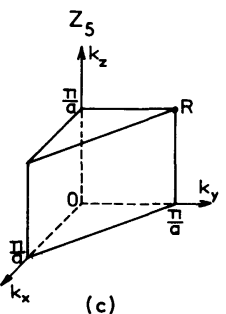

(c)

FIG. 3. - The different Brillouin zones of a magnetic sublattice corresponding to the three antiferromagnetic structures under investigation. In fact all these Brillouin zones being symmetrical with respect to the three coordinate planes, only the parts in the first octant are represented.

In the limit where $H_{a} \rightarrow 0$, it is easy to see from expressions (47) that $\lambda_{\mathbf{k}}$ and $\mu_{\mathbf{k}}$ remain positive for all $\mathbf{k}$ if, and only if, both $J_{\|}$and $J_{\perp}$ are positive, a result which confirms the classical predictions.

Typical dispersion curves corresponding to relations (47) when $H_{a} \rightarrow 0$ are represented in figure $4 b$ for $\mathbf{k} /[001]$ and $\mathbf{k} / /[100]$. In the (110) plane $\lambda_{\mathbf{k}}$ and $\mu_{\mathbf{k}}$ are degenerate, but outside this plane the degeneracy is removed giving two modes. This result was already obtained [10] in the calculation of the spin wave spectrum of $\mathrm{KCoF}_{3}$ which corresponds to this configuration and to this kind of Hamiltonian.

3.2 Configuration $Z_{2}$. - Each magnetic sublattice is a simple tetragonal lattice, with a unit cell of sides $(a, a, 2 a)$. The Brillouin zone is represented in figure $3 b$. The Hamiltonian (38) is such that

$$
\begin{aligned}
\omega_{\mathbf{k}} & =2 S\left(-2 J_{\perp}+J_{\|}\right)+S\left(J_{\perp}+J_{\|}\right) \times \\
& \times\left(\cos k_{x} a+\cos k_{y} a\right)+H_{a}, \\
\gamma_{\mathbf{k}} & =\frac{S}{2}\left(J_{\|}-J_{\perp}\right)\left(\cos k_{x} a-\cos k_{y} a\right), \\
\delta_{\mathbf{k}} & =2 S J_{\perp} \cos k_{z} a, \\
\varepsilon_{\mathbf{k}} & =0 .
\end{aligned}
$$

From (43) and (48) the dispersion relations are given by

$$
\begin{gathered}
\lambda_{\mathbf{k}}=4 S\left|J_{\perp}\right|\left[\left(-t \cos ^{2} \alpha+\sin ^{2} \beta+\sin ^{2} \gamma-\frac{H_{a}}{4 S J_{\perp}}\right)\left(\sin ^{2} \alpha-t \cos ^{2} \beta+\cos ^{2} \gamma-\frac{H_{a}}{4 S J_{\perp}}\right)\right]^{1 / 2}, \\
\begin{aligned}
\mu_{\mathbf{k}}=4 S\left|J_{\perp}\right|\left[\left(-t \cos ^{2} \alpha+\sin ^{2} \beta+\cos ^{2} \gamma-\frac{H_{a}}{4 S J_{\perp}}\right)\left(\sin ^{2} \alpha-t \cos ^{2} \beta+\sin ^{2} \gamma-\frac{H_{a}}{4 S J_{\perp}}\right)\right]^{1 / 2}, \\
-\frac{\pi}{2}<\alpha, \beta \leqslant \frac{\pi}{2} \quad \text { and } \quad-\frac{\pi}{4}<\gamma \leqslant \frac{\pi}{4} .
\end{aligned}
\end{gathered}
$$

with

The magnetic stability is assured for $J_{\perp}<0$ and $J_{\|}>0$, in accordance with the classical prediction.

3. 3 Configuration $Z_{5}$. - Each magnetic sublattice is a simple tetragonal lattice rotated by $\pi / 4$ around the $z$ axis with respect to the fourfold axes of the cubic lattice. The first Brillouin zone is given in figure $3 c$. The parameters involved in the Hamiltonian are

$$
\begin{aligned}
\omega_{\mathbf{k}} & =2 S\left(2 J_{\perp}-J_{\|}\right)+2 S J_{\perp} \cos k_{z} a+H_{a}, \\
\gamma_{\mathbf{k}} & =0, \\
\delta_{\mathbf{k}} & =S\left(J_{\|}+J_{\perp}\right)\left(\cos k_{x} a+\cos k_{y} a\right), \\
\varepsilon_{\mathbf{k}} & =S\left(J_{\|}-J_{\perp}\right)\left(\cos k_{x} a-\cos k_{y} a\right) .
\end{aligned}
$$

From (43) and (50) the dispersion relations $\lambda_{\mathbf{k}}$ and $\mu_{\mathbf{k}}$ are given by 


$$
\begin{aligned}
& \lambda_{\mathbf{k}}=4 S\left|J_{\perp}\right|\left[\left(\sin ^{2} \alpha-t \cos ^{2} \beta+\cos ^{2} \gamma+\frac{H_{a}}{4 S J_{\perp}}\right)\left(-t \sin ^{2} \alpha+\cos ^{2} \beta+\cos ^{2} \gamma+\frac{H_{a}}{4 S J_{\perp}}\right)\right]^{1 / 2}, \\
& \mu_{\mathbf{k}}=4 S\left|J_{\perp}\right|\left[\left(\cos ^{2} \alpha-t \sin ^{2} \beta+\cos ^{2} \gamma+\frac{H_{a}}{4 S J_{\perp}}\right)\left(-t \cos ^{2} \alpha+\sin ^{2} \beta+\cos ^{2} \gamma+\frac{H_{a}}{4 S J_{\perp}}\right)\right]^{1 / 2},
\end{aligned}
$$

with

$$
-\frac{\pi}{2}<\alpha, \beta, \gamma \leqslant \frac{\pi}{2} \quad \text { and } \quad|\alpha|+|\beta| \leqslant \frac{\pi}{2} .
$$

The magnetic stability is assured when $J_{\perp}>0$ and $J_{\|}<0$, still in accordance with the classical prediction of section 2 .

In fact, the dispersion relations (49) and (51) of $Z_{2}$ and $Z_{5}$ could have been deduced from the dispersion relations (33) and (47) of $Z_{1}$ and $Z_{8}$ following remarks in section 2.4 and the transformation (25). For example an excitation of $Z_{5}$ is obtained from an excitation of $Z_{1}$ by changing $J_{\perp}$ into $-J_{\perp}$ (or $t$ into $-t$ ) and by taking account of the new spin configuration expressed by (23). More precisely as we have

$$
\begin{aligned}
& S_{i}^{x}\left(\mathrm{Z}_{5}\right)=\mathrm{e}^{i \mathbf{k}^{x} \cdot \mathbf{R}_{i}} \cdot S_{i}^{x}\left(\mathrm{Z}_{1}\right), \\
& S_{i}^{y}\left(\mathrm{Z}_{5}\right)=\mathrm{e}^{i \mathbf{k}^{\boldsymbol{y}} \cdot \mathbf{R}_{i}} \cdot S_{i}^{y}\left(\mathrm{Z}_{1}\right),
\end{aligned}
$$

$\mathbf{k}^{x}$ and $\mathbf{k}^{y}$ being defined by (22), a spin wave with a wave vector $\mathbf{k}$ in $Z_{1}$ is replaced by a spin wave of wave vector $\mathbf{k}+\mathbf{k}^{x}$ and $\mathbf{k}+\mathbf{k}^{y}$ in $Z_{5}$. So the dispersion relations (51) are simply obtained from (33) by replacing

$$
t, \alpha, \beta, \gamma \quad \text { by } \quad-t, \alpha, \beta+\frac{\pi}{2}, \gamma+\frac{\pi}{2}
$$

for one branch and

$$
t, \alpha, \beta, \gamma \quad \text { by } \quad-t, \alpha+\frac{\pi}{2}, \beta, \gamma+\frac{\pi}{2}
$$

for the other branch.

It is thus not necessary to draw the dispersion curves of $Z_{5}$ as they are not fundamentally different from those of $Z_{1}$. Only the geometry of the Brillouin zone has changed.

We must note that the point $0(k=0)$ in $Z_{1}$ for which the excitation energy $\lambda$ is zero in the frame of the linearized Holstein-Primakoff formalism is equivalent to the point $\mathrm{R}$ with $\mathbf{k}(0, \pi / a, \pi / a)$ at the surface of the Brillouin zone (and to the equivalent points) of $Z_{5}$ where $\lambda_{\mathbf{k}}=0$.

Similarly we could deduce the dispersion relations of $\mathrm{Z}_{2}$ from those of $\mathrm{Z}_{8}$ with the same transformation (53). However, it is not obvious how to deduce a priori which of the transformations (53) is associated with $\lambda$ and $\mu$, and this is the reason why the dispersion relations of $Z_{2}$ were obtained from the general formulas (43). Still in this case the central point 0 of the Brillouin zone of $Z_{8}$ is equivalent to the points
$(0, \pi / a, 0)$ and $(\pi / a, 0,0)$ at the surface of the Brillouin zone of $Z_{2}$ where $\lambda_{k}$ (or $\mu_{k}$ ) given by (49) vanish.

To summarize, we have shown that, in the framework of our approximation, the four configurations represented in figure 1 obtained classically, are stable with respect to the magnetic spin wave excitations with the same range of validity for the two parameters $J_{\|}$and $J_{\perp}$. For the special cases where $J_{\|}$or $J_{\perp}$ are zero, one of the excitation energies is zero on a whole line or a whole plane of the Brillouin zone. This is in agreement with the high degeneracy of the classical ground state as shown in the previous section.

Finally we would like to point out that our results are not in contradiction with the Goldstone theorem [11] which may be summarized in the following way [12] : when a system has a continuous broken symmetry with short range interactions, one branch of the elementary excitations from a particular ground state is such that $\lambda(\mathbf{k}) \rightarrow 0$ when $\mathbf{k} \rightarrow 0$, i.e. there is no gap at the origin. We have already said that for the configuration $Z_{1}, \lambda_{k}$, as given by equation (33), has no gap at the origin (see Fig. 4a). But as shown in reference [1] the quantum ground state energy $E_{0}^{\prime}$ defined by (2) is anisotropic for $J_{\|} \neq J_{\perp}$, the easy direction of magnetization being a fourfold axis of the cube. Thus there is no continuous group of transformations leaving the exchange Hamiltonian (4) invariant. We can then expect a gap in the dispersion relations for $\mathbf{k}=0$. In fact there is a gap in the spin wave spectrum which comes from higher order terms in the expansion of the spin components in terms of creation and annihilation operators. Including all the terms of the 4 th order in the Bose operators we obtained a gap $\Delta \lambda$ at the origin for the $Z_{1}$ configuration which is given by

$$
\begin{aligned}
\Delta \lambda(\mathbf{k}= & 0)=\frac{\left(J_{\|}-J_{\perp}\right)^{2}}{4 N\left|J_{\perp}\right|} \times \\
& \times \sum_{\mathbf{k}^{\prime}} \frac{\left(\cos k_{x}^{\prime} a-\cos k_{y}^{\prime} a\right)^{2}}{3-\cos k_{x}^{\prime} a-\cos k_{y}^{\prime} a-\cos k_{z}^{\prime} a}
\end{aligned}
$$

where the summation over $\mathbf{k}^{\prime}$ is taken over the first Brillouin zone. We see from (54) that there is no gap only if $J_{\|}=J_{\perp}$ (Heisenberg ferromagnet case). Furthermore this gap at the origin insures the stability of $Z_{1}$ and consequently of $Z_{5}$.

4. Quantum ground state energy. - We have shown in the previous section that the ground state energy $E_{0}^{\prime}$ 
(a): $Z_{1}$
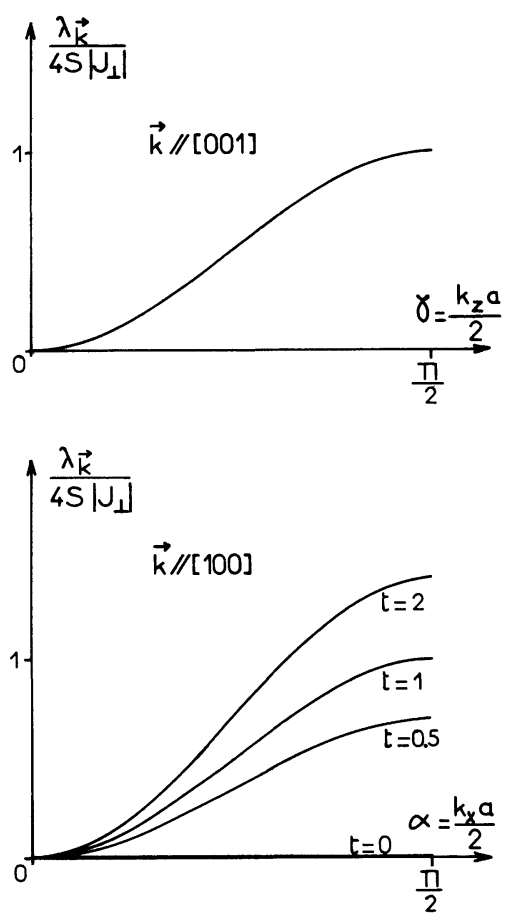

(b): $\mathbf{z}_{\mathbf{8}}$
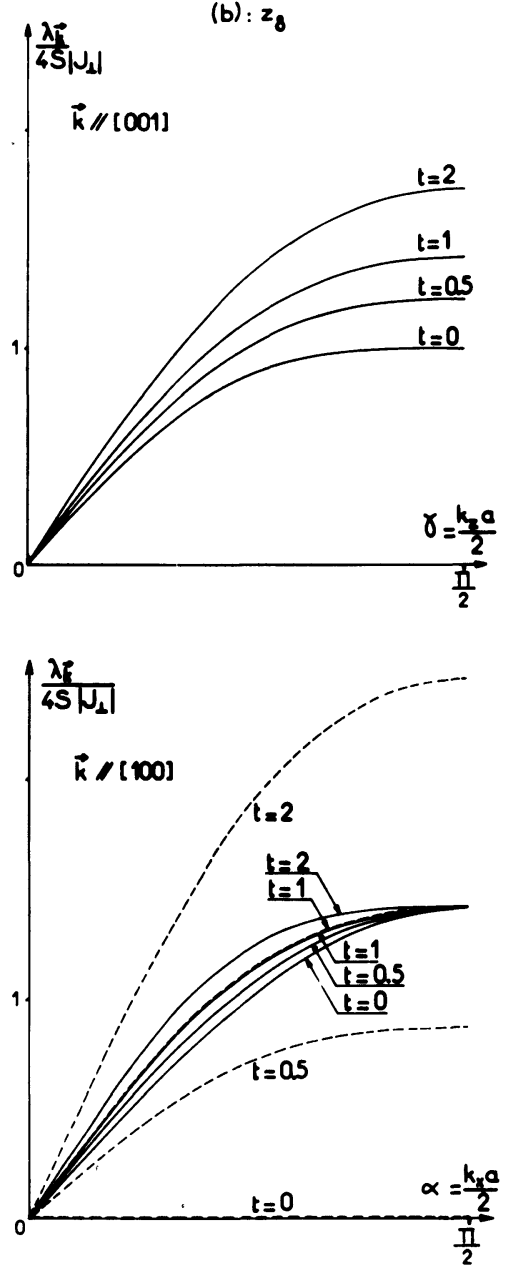

Fig. 4. - Magnetic dispersion curves for the configurations a) $\left.\mathrm{Z}_{1}, b\right) \mathrm{Z}_{8}$, for $H_{a}=0$ and $\mathbf{k}$ parallel to the fourfold axes. Broken lines correspond to $\mu_{\mathbf{k}}$ when different from $\lambda_{\mathbf{k}}$. corresponding to the Hamiltonian (38) is given by equation (44) where $E_{0}$ is the classical ground state energy and $\frac{1}{2} \sum_{\mathbf{k}}\left(\lambda_{\mathbf{k}}+\mu_{\mathbf{k}}-2 \omega_{\mathbf{k}}\right)$ is the quantum mechanical zero point energy which comes from the fact that we have assumed only an approximate ground state. First it must be noted from the Bogolyubov transformation (see expressions (45) of $u_{\mathbf{k}}$ and $u_{\mathbf{k}}^{\prime}$ ) that $\lambda_{\mathbf{k}} \leqslant \omega_{\mathbf{k}}+\varepsilon_{\mathbf{k}}$ and $\mu_{\mathbf{k}} \leqslant \omega_{\mathbf{k}}-\varepsilon_{\mathbf{k}}$, thus we have always $E_{0}^{\prime}<E_{0}$.

Taking into account the discussion of section 2, we know that $\left|E_{0}^{\prime}\right|$ is the same for $Z_{1}$ and $Z_{5}$ on one side and for $Z_{2}$ and $Z_{8}$ on the other side. So we can restrict ourselves to $Z_{1}$ and $Z_{8}$.

It is easy to show that for $Z_{8}$, we have from (46), in the limit where $H_{a}=0$,

$$
E_{0}^{\prime}=-N S(S+1) J_{\perp}(t+2)+\frac{1}{2} \sum_{\mathbf{k}}\left(\lambda_{\mathbf{k}}+\mu_{\mathbf{k}}\right) \text {. }
$$

The corresponding expression for $Z_{1}$ was given by equation (36) of reference [1].

In figure 5 we show the variation of $E_{0}^{\prime} /\left(N J_{\perp}\right)$ for $S=\frac{1}{2}$ and $H_{a}=0$, as a function of $t$, after numerical integration over the respective Brillouin zones.

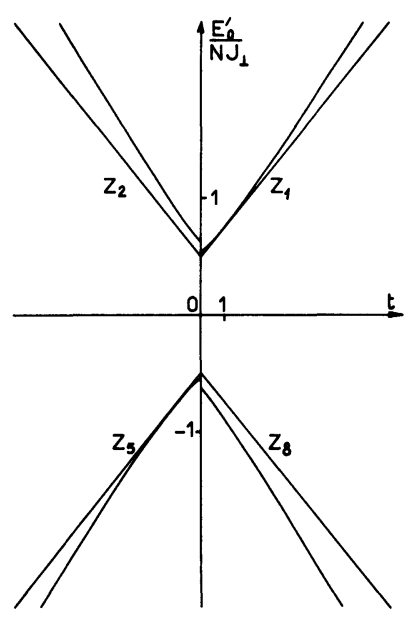

FIG. 5. - Variation of the ground state energy as a function of $t$ : the curves represent $E_{0}^{\prime} /\left(N J_{\perp}\right)$ given by $(55)$ and the straight lines represent the classical energy $E_{0} /\left(N J_{\perp}\right)$ given by (24). In agreement with figure 2 , when $J_{\perp}>0, Z_{8}$ and $Z_{5}$ are the ground configurations for $t>0$ and $t<0$ respectively, and when $J_{1}<0, Z_{1}$ and $Z_{2}$ are the ground configurations for $t>0$ and $t<0$ respectively.

Finally, for $Z_{8}$ in order to get an estimation of the discrepancy between our approximate ground state $|f\rangle$ obtained through the Bogolyubov transformation when there are no spin wave excitations, and the classical ground state, it is interesting to calculate the mean spin deviation $\left\langle n_{i}\right\rangle$ in one sublattice which is given by

$$
\left\langle n_{i}\right\rangle=\frac{2}{N} \sum_{\mathbf{k}}\left\langle f\left|a_{\mathbf{k}}^{\dagger} a_{\mathbf{k}}\right| f\right\rangle,
$$


where $a_{\mathbf{k}}$ and $a_{\mathbf{k}}^{\dagger}$ are defined by (37). From the inverse linear transformation (39) we get

$$
\left\langle n_{i}\right\rangle=\frac{2}{N} \sum_{\mathbf{k}}\left(u_{\mathbf{k}}^{2}+u_{\mathbf{k}}^{\prime 2}\right) .
$$

Using the expressions of $u_{\mathbf{k}}$ and $u_{\mathbf{k}}^{\prime}$ given by (45) we obtain

$$
\left\langle n_{i}\right\rangle=\frac{2}{N} \sum_{\mathbf{k}}\left(\frac{\omega_{\mathbf{k}}+\varepsilon_{\mathbf{k}}}{4 \lambda_{\mathbf{k}}}+\frac{\omega_{\mathbf{k}}-\varepsilon_{\mathbf{k}}}{4 \mu_{\mathbf{k}}}-\frac{1}{2}\right) .
$$

$\left\langle n_{i}\right\rangle$ has been calculated by numerical integration over the Brillouin zone for various values of the parameter $t$. The results are shown in figure 6 , together with the corresponding ones obtained for $Z_{1}[1]$.

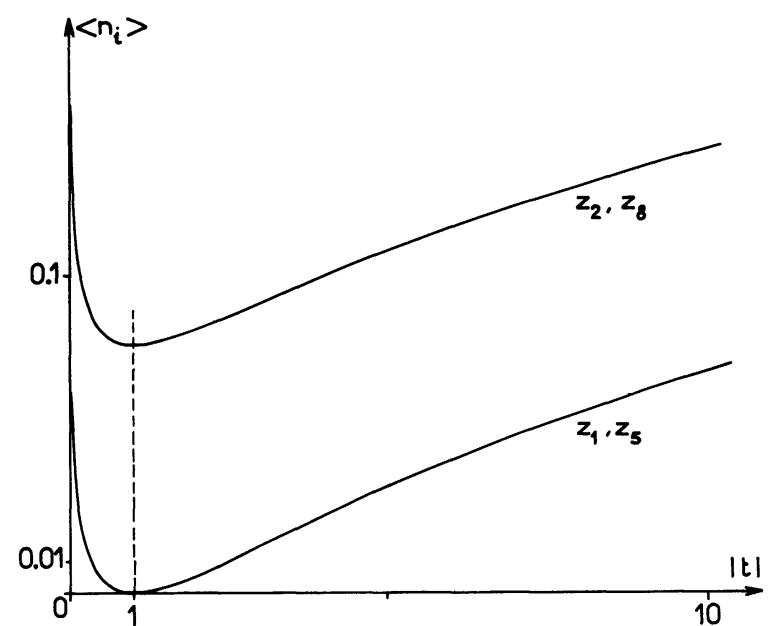

FIG. 6. - Variation of the mean spin deviation $\left\langle n_{i}\right\rangle$ as a function of $|t|$ for the various configurations.

5. Conclusion. - The fact that $Z_{5}$ may be obtained from $Z_{1}$ by changing $J_{\perp}$ into $-J_{\perp}$ and by using the local axes defined by $(25)$ has a very striking consequence. Since we know that for $J_{\|}=J_{\perp}$ the configuration $Z_{1}$ is an eigenstate of the exchange Hamiltonian (Heisenberg ferromagnet) we must have the same property for $Z_{5}$ when $J_{\|}=-J_{\perp}$. Indeed this can be very easily proved in the following way : we start from the quantum ground state $|f\rangle$ with no spin deviation in each sublattice. This means that

$$
|f\rangle=\prod_{i}\left|S_{i z}=\frac{1}{2}\right\rangle \prod_{j}\left|S_{j z}=-\frac{1}{2}\right\rangle,
$$

where $i$ and $j$ refer to the spins of the two sublattices respectively like in figure $1 d$. It is easy to check that for $J_{\|}=-J_{\perp}$ we have

$$
\cdot \mathcal{H}|f\rangle=-3 N S^{2} J_{\perp}|f\rangle,
$$

where $\mathcal{H}$ is the exchange Hamiltonian of the problem and is given by (36) with $H_{a}=0$. Thus $|f\rangle$ is an eigenstate of $\mathcal{H}$. It must be pointed out that this feature is specific to the configuration $Z_{5}$ for $t=-1$ and that no equivalent situation arises for the two other antiferromagnetic configurations. To our knowledge, apart from the Ising model, this is the only known case of three dimensional two sublattice antiferromagnet for which the ground state is perfectly known [13].

Another point is that for $Z_{2}$ and $Z_{8},\left\langle n_{i}\right\rangle$ is minimum for $|t|=1$ as can be seen in figure 6 . In this case $\left\langle n_{i}\right\rangle=0.08$, a very well known result [14] for the Heisenberg antiferromagnet.

Finally we shall conclude that the phase diagram obtained from the quantum energy ground state $E_{0}^{\prime}$ and represented in figure 5 is not essentially different from the classical phase diagram. The quantum theory predicts the same ground configuration as the classical theory for any values of the couple of parameters $J_{\|}$and $J_{\perp}$. The discontinuity which appears for $t=0\left(J_{\|}=0\right)$ on $E_{0}^{\prime}$ is not very significant because, first of all, our dispersion relations are strictly speaking not valid for this value except if we add an anisotropy field, and because $\left\langle n_{i}\right\rangle$ is respectively equal to 0.063 and 0.153 for $Z_{1}$ and $Z_{8}$, so that the approximate ground states are rather poorly defined. The same difficulties arise for $|t|=\infty\left(J_{\perp}=0\right)$ and they are essentially due to the high degeneracy of the ground state which was underlined in the classical discussion.

Acknowledgments. - We are indebted to Prs. P. Nozières, P. M. Levy and P. Averbuch for stimulating discussions, and to Pr. R. A. B. Devine for a critical reading of the manuscript.

\section{References}

[1] Belorizky, E., Casalegno, R. and Fries, P., Phys. Status Solidi (b) 77 (1976) 495.

[2] Abragam, A. and Bleaney, B., Electron Paramagnetic Resonance of Transition Ions (Clarendon Press, Oxford) 1969.

[3] Levy, P. M., Magnetic Oxides, edited by D. J. Craik (Wiley, London) 1975, chap. 4.

[4] Wolf, W. P., J. Physique Colloq. 32 (1971) C1-26.

[5] Yoshimori, A., J. Phys. Soc. Jpn. Vol. 14, no 6 (1959) 807.

[6] Luttinger, J. M., Phys. Rev. 81 (1951) 1015.

[7] Lutringer, J. M. and Tisza, L., Phys. Rev. 70 (1946) 954.

[8] Tinkham, M., Group Theory and Quantum Mechanics (Mc Graw Hill Book Company, New York) 1964.
[9] KOSTER, G. F., Space groups and their representations, Solid Stute Phis. 5 (1957) 173.

[10] Buyers, W. J. L., Holden, T. M., Svensson, E. C., Cowley, R. A. and Hutchings, M. T., J. Phys. C 4 (1971) 2139.

[11] Lange, R. V., Phys. Rev. 146, no 1 (1966) 301.

[12] Sivardiere, J., Séminaires Daniel Dautreppe, Université de Grenoble (1976). Private communication.

[13] Belorizky, E., Casalegno, R. and Niez, J. J., Phys. Lett. A 65A, n 4 (1978) 340.

[14] Herpin, A., Théorie du Magnétisme (Presses Universitaires de France, Paris) 1968. 